\title{
Strongly Accelerated and Humidity-Independent Drying of Nanochannels Induced by Sharp Corners
}

\author{
J.C. T. Eijkel, B. Dan, ${ }^{*}$ H. W. Reemeijer, D. C. Hermes, J. G. Bomer, and A. van den Berg \\ BIOS/Lab-on-a-Chip Group, MESA+Research Institute, University of Twente, Enschede, The Netherlands
}

(Received 7 June 2005; published 16 December 2005)

\begin{abstract}
Measurements are shown indicating that the drying rate of nanochannels can be enhanced by up to 3 orders of magnitude relative to drying by vapor diffusion, and that the drying rate is independent of the relative humidity of the environment up to a relative humidity of more than $90 \%$. Micromachined Pyrex glass nanochannels of $72 \mathrm{~nm}$ height and with sharp corners (corner angles 7 degrees) were used. Available theory shows that the sharp corners function as a low-resistance pathway for liquid water, siphoning (wicking) the water to a location close to the channel exit before it evaporates. The described phenomena are of importance for the understanding of drying processes in industry and agriculture. The introduction of sharp corners or grooves can furthermore be beneficial for the functioning of microheat pipes and capillary-pumped loops.
\end{abstract}

DOI: 10.1103/PhysRevLett.95.256107

PACS numbers: 68.03.Fg

Understanding the drying mechanism of porous materials is of importance in many industries such as the food, paper, pharmaceutical, and textile industry [1-3]. It has previously been observed that microporous media dried approximately 1 order of magnitude faster than can be expected from vapor diffusion alone [4,5]. Flow in liquid water films held on surfaces and flow of water held in corners or grooves were thought to cause this acceleration [6]. The contribution of film flow to drying has been experimentally investigated in cylindrical nanocapillaries, where it caused a tenfold increase of drying rate, [7] but the contribution of corner flow has never been investigated. Here we report on experiments using noncylindrical micromachined nanochannels to quantify corner flow.

Drying results from three water transport mechanisms: vapor diffusion, film flow, and corner flow. To specifically investigate corner flow we designed an array of high aspect ratio (width $\gg$ height) noncylindrical channels of equal height but different width (Fig. 1). The three water transport mechanisms schematically are shown in Fig. 2. When corner flow dominates the drying process in a channel, the drying rate will depend on the inverse of the channel width, because the number of corners is independent of width but the total water volume inside the channel proportional with width. Drying due to film flow (for width $\gg$ height) and vapor diffusion in contrast does not depend on the channel width. Arrays of Pyrex channels, open on two sides, were manufactured in a clean room. Channels were wet etched (hydrofluoric acid) into one Pyrex wafer using a photolithographic mask. This wafer was bonded by thermal fusion to a second wafer which had access holes for filling. The channels were $4 \mathrm{~mm}$ long, $72.4 \pm 0.8 \mathrm{~nm}$ high (determined by AFM) and the width in the array differed from 2 to $30 \mu \mathrm{m}$. The channel shape was an isosceles trapezoid of very high aspect ratio (width/height $>40$ ) (Fig. 1 bottom). The angle of the sharp corner was determined to be $6.6 \pm 0.7$ degrees by SEM measurements of bonded chips and by AFM measurements prior to bonding of the Pyrex plates. The sharp corners are thought to be created by underetching of the lithographic mask. Directly after production, the chips were stored individually wrapped in aluminum foil to prevent atmospheric fouling. The water contact angle was determined from the filling speed of the channels by fitting the Washburn equation [8] accounting for a slightly (17\%) increased viscosity as recently found for capillary filling of water in nanochannels [9] The average contact angle was found to be $34^{\circ}(\cos \theta=0.83)$.

Drying experiments were performed in a chamber in which the relative humidity (RH) was controlled by equilibration with different saturated salt solutions. The RH was measured using a commercially available sensor, HIH3602 -A (Honeywell) with an accuracy of $\pm 2 \% \mathrm{RH}$ at

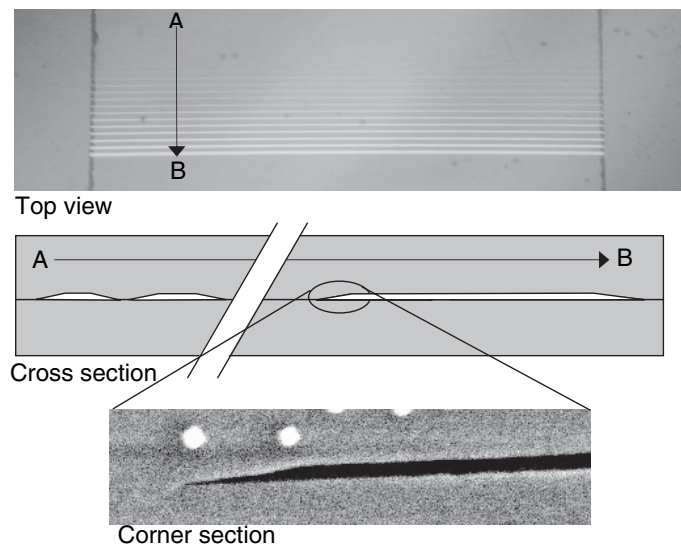

FIG. 1. Top: Microphotograph of an array of parallel $72 \mathrm{~nm}$ high and $4 \mathrm{~mm}$ long Pyrex nanochannels of varying width (30 to $2 \mu \mathrm{m})$. The channels are the white lines running from left to right and are open to the atmosphere at the sides. Middle: schematic cross section through the device along the line $A-B$. Bottom: SEM photograph of a cross section of the corner section, showing the $7^{\circ}$ corner angle. 


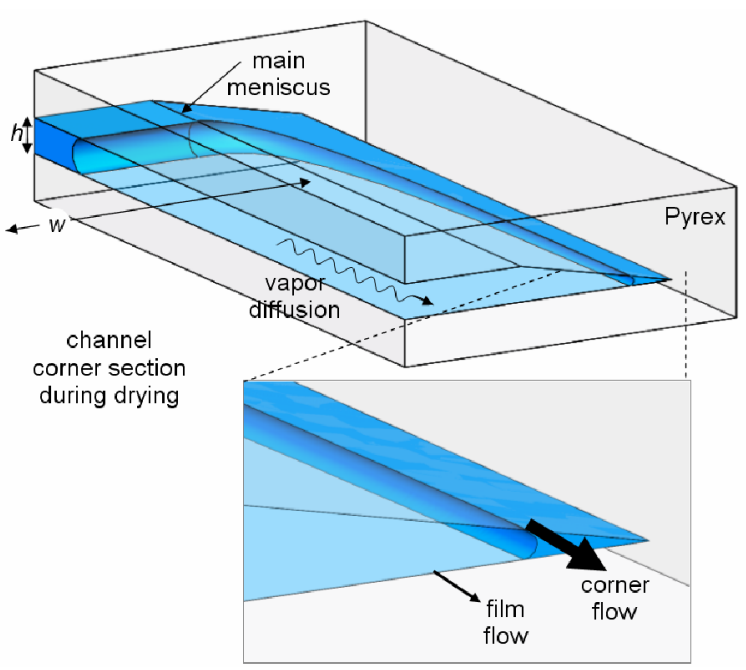

FIG. 2 (color online). The corner section of a nanochannel during drying. Three processes, namely, vapor diffusion, film flow, and corner flow, contribute to the water flux out of the channel and make the main meniscus move backwards. Channel height $h$ and width $w$ are indicated.

$25^{\circ}$. Measurement of the drying rate proceeded by first depositing a drop of demineralized water (specific resistance $18 \mathrm{MOhm} \mathrm{cm}$ ) at the channel openings on one side, causing a rapid filling of the channels by capillarity. Subsequently, the chip was placed in the RH chamber for equilibration. When the desired stable humidity condition was reached, the excess water was removed by suction without opening the chamber, leaving only the water inside the channels and starting the drying process. Drying was recorded using a video camera. The temperature inside the chamber was not controlled. Temperatures in different experiments varied between 18.5 and $23.7^{\circ} \mathrm{C}$, and during every experiment the temperature remained constant within $0.5^{\circ} \mathrm{C}$.

Figure 3 shows a snapshot of a channel array during the drying process. Narrow channels are seen to dry faster than wide ones indicating the predominance of corner flow. In Fig. 4 the measured drying rate of the channels of one array at a $\mathrm{RH}$ of $83 \%$ is plotted against the reciprocal of the channel width, $w^{-1}$. A clear dependence on channel width is seen, indicating the predominance of corner flow [see Eq. (1)]. The data coincide satisfactorily with the prediction from the model presented below. To illustrate the acceleration of the drying process, the expected drying rates for drying by vapor diffusion alone and by a combination of vapor diffusion and film flow are also shown in Fig. 4. Drying is accelerated by a factor of 50-500, indicating the efficiency of the corner flow as transport mechanism. A total of 31 experiments were thus performed, each on a different channel array and measuring at different RH. Of these, 15 experiments had to be discarded because during drying the meniscus stayed pinned at the channel exit on one side, or because patchwise drying occurred in the entire channel. For the remaining

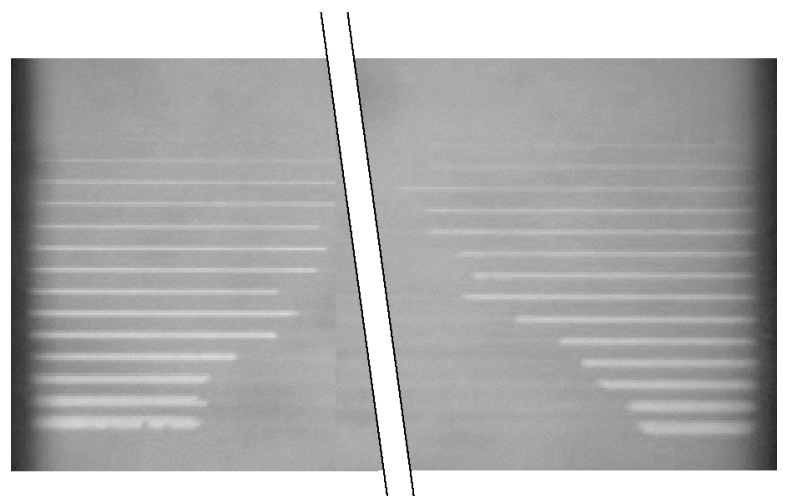

FIG. 3. Snapshot taken $27 \mathrm{~s}$ into the drying of a channel array at a $\mathrm{RH}$ of $91.8 \%$. Channel sections that have dried up appear white on the photo. The $4 \mathrm{~mm}$ long channel dries from both ends; the middle part of the array has been omitted for clear viewing.

16 experiments the drying rates were determined by plotting the dried-up length squared against time. Thirteen experiments in this way yielded significant evidence for the predominance of corner flow. When corner flow dominates, the theoretical analysis presented beneath predicts that the drying rate will be virtually independent of RH up to high RH, and this indeed was experimentally confirmed. Figure 5 shows the observed drying rates for channels of $16 \mu \mathrm{m}$ width plotted as a function of RH. The drying rate is independent of $\mathrm{RH}$ up to $\mathrm{RH}=93 \%$, and the observed rate coincides satisfactorily with the model prediction.

For theoretical modeling we assumed that vapor diffusion, film flow, and corner flow contribute to the drying process (Fig. 2). The three resulting fluxes are coupled by assuming equilibrium between water vapor and liquid water in films and corners at any point along the drying

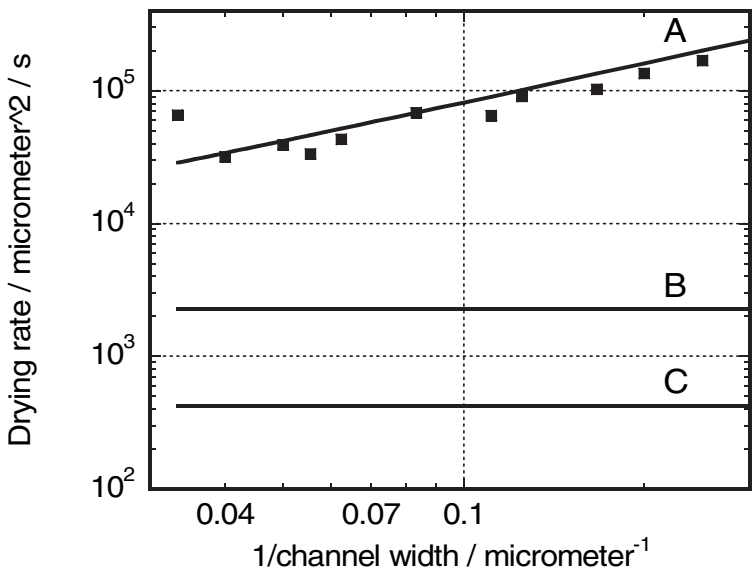

FIG. 4. Observed drying rates ( $\square$ ) as a function of inverse channel width at $\mathrm{RH}=83 \%$. The linear dependence on 1 /width indicates a predominance of drying by corner flow [compare Eq. (1)]. Shown as lines are the theoretical drying rates for drying by (A) corner flow, film flow, and vapor diffusion, (B) film flow and vapor diffusion, and $(\mathrm{C})$ vapor diffusion only. The observed drying rates are 50-500 times faster than the theoretical drying rate by vapor diffusion alone. 


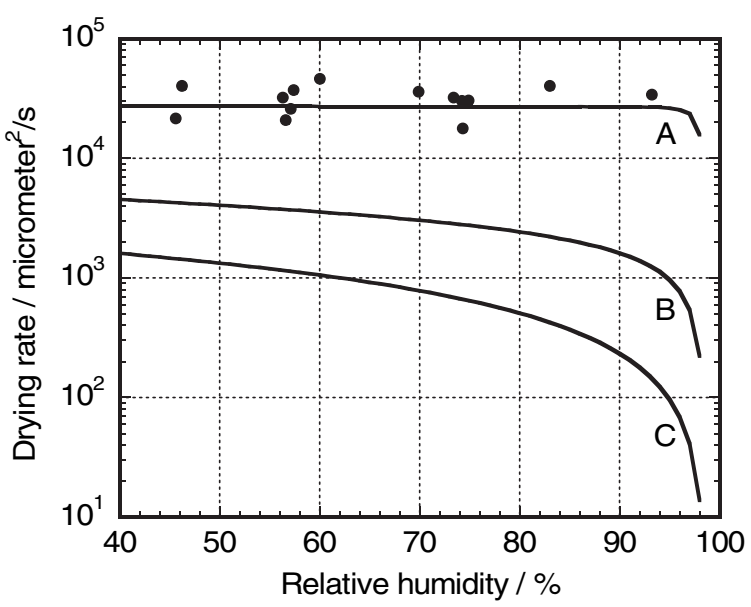

FIG. 5. Observed drying rates (-) in $16 \mu \mathrm{m}$ wide channels as a function of RH. Drying is independent of relative humidity up to $\mathrm{RH}=93 \%$. Also shown are the theoretical drying rates for drying (A) by corner flow, film flow, and vapor diffusion, (B) by film flow and vapor diffusion, and $(\mathrm{C})$ by vapor diffusion only.

axis $[7,10]$. The local water chemical potential per unit volume $\Psi(\mathrm{Pa})$ is related to the $\mathrm{RH}$ and to the radius of the corner meniscus by the Kelvin equation [11,12]

$$
\Psi=\frac{R T}{V_{w}} \ln \left(\frac{p}{p_{\text {sat }}}\right)=\frac{\gamma \cos \theta}{r},
$$

and to the adsorbed film thickness by the Lifshitz theory for the action of dispersion forces $[7,10]$

$$
f=\sqrt[3]{\left(\frac{A_{h}}{6 \pi \Psi}\right)}
$$

Here $V_{w}$ is the molar volume of water $(18 \times$ $\left.10^{-6} \mathrm{~m}^{3} / \mathrm{mol}\right), p$ the water vapor pressure $(\mathrm{Pa}), p_{\text {sat }}$ the saturated water vapor pressure, $\gamma$ the water surface tension $(0.072 \mathrm{~N} / \mathrm{m}), \theta$ the water/Pyrex contact angle, $r$ the meniscus radius (negative if concave), and $A_{h}$ the Hamaker constant, for which we assume the value of $-1.9 \times$ $10^{-19} \mathrm{~J}[13]$.

The system is essentially isothermal, considering the minute water quantities concerned, obviating the need to include the influence of temperature gradients. The three coupled fluxes $J_{v}, J_{f}$, and $J_{c}$, [ $\left.\mathrm{m}^{3} \operatorname{liquid} /\left(\mathrm{m}^{2} \mathrm{~s}\right)\right]$, respectively, from vapor diffusion, film flow and corner flow as a function of $\Psi$ are

$$
\begin{aligned}
J_{v} & =D \frac{V_{w} p_{\text {sat }}}{R T}\left(-\frac{d \exp \left(\Psi V_{w} / R T\right)}{d x}\right), \\
J_{f} & =\frac{2 A_{h}}{18 \pi \eta h \Psi}\left(-\frac{d \Psi}{d x}\right) \\
\text { and } J_{c} & =\underbrace{\left[\frac{1}{\beta}\left(\frac{2}{\tan (\alpha / 2)}-\pi+\alpha\right)\right]}_{\text {A }} \frac{r^{4}(x)}{w h \eta}\left(-\frac{d \Psi}{d x}\right) .
\end{aligned}
$$

Here $D$ is the vapor diffusion coefficient $\left(\mathrm{m}^{2} / \mathrm{s}\right)$ for which a value of $1.5 \times 10^{-5} \mathrm{~m}^{2} / \mathrm{s}$ is assumed since the Knudsen number is about 3, $\eta$ the water viscosity (the bulk viscosity value of $10^{-3} \mathrm{Pas}$ is assumed), $\beta$ a dimensionless resistance parameter characteristic for the corner geometry [14], and $\alpha$ the corner angle (radians). For $\theta$ the average value experimentally determined from capillary filling $\left(34^{\circ}\right)$ was used. The magnitude of the corner flow is strongly determined by the value of the term $A$, which includes both $\beta$ and a geometrical term. The amount of water held in a $6.6^{\circ}$ corner at any meniscus radius (determined by the local RH) for geometric reasons is about 70 times larger than in a $90^{\circ}$ corner. In addition, $\beta$ is about 10 times smaller in the sharp corners since the liquid on average is further removed from the wall [14]. Because of both effects $A$ (taken for two corners) increases from 0.0047 at $\alpha=90^{\circ}$ to 3.38 at $\alpha=6.6^{\circ}$, a factor of 720 . Only the two sharp corners were considered per channel, since virtually no water is held in the two blunt ones.

The coupled flux system can be solved in a first-order approximation by the assumption that the sum of the three water fluxes is constant along the axial direction, producing the total drying flux $J\left[\mathrm{~m}^{3} /\left(\mathrm{m}^{2} \mathrm{~s}\right)\right]$ as a function of $\Psi_{0}$ (at the main meniscus and determined by its curvature) and $\Psi_{e}$ (at the channel exit and determined by the environmental RH). Subsequent time integration of $J$ (taking into account simultaneous drying from both channel ends) gives for the drying rate $L^{2} / t$ (with 1 the one-sided dried-up channel length and $L=21$ )

$$
\frac{L^{2}}{t}=\frac{(2 l)^{2}}{t}=C_{v}+\frac{C_{f}}{h}+\frac{C_{c}}{w h}
$$

where

$$
\begin{aligned}
C_{v}= & \frac{8 D V_{w} P_{\text {sat }}}{R T}\left[\exp \left(\frac{\Psi_{0} V_{w}}{R T}\right)-\exp \left(\frac{\Psi_{e} V_{w}}{R T}\right)\right], \\
C_{f}= & \frac{-16 A}{18 \pi \eta} \ln \left(\frac{\Psi_{e}}{\Psi_{0}}\right) \\
\text { and } \quad C_{c}= & 8\left[\frac{1}{\beta}\left(\frac{2}{\tan (\alpha / 2)}-\pi+\alpha\right)\right] \\
& \frac{(\gamma \cos \theta)^{4}}{\eta}\left(\frac{1}{\Psi_{e}^{3}}-\frac{1}{\Psi_{0}^{3}}\right) .
\end{aligned}
$$

The first obvious implication of Eq. (1) is that nanochannels are very suitable to demonstrate drying by film flow and corner flow, since the contributions of both liquid film flow and liquid corner flow to the drying rate are proportional to the inverse of the channel height. The second implication is that only the contribution of the corner flow depends on the channel width $w$, and is proportional to $w^{-1}$.

As shown, the theoretical predictions coincide well with the measurement results, both for the relation between drying rate and channel width (Fig. 4) and between drying rate and RH (Fig. 5). The counterintuitive observation that the drying rate is virtually independent of $\mathrm{RH}$ can be 
understood by inspecting $C_{c}$ in Eq. (2). $C_{c}$, and therefore the drying rate in a corner-flow dominated system, will be practically independent of $\Psi_{e}$ (determined by the environmental RH) because both $\Psi_{e}$ and $\Psi_{0}$ occur as the inverse third power in the bracketed term and $\left|\Psi_{e}\right| \gg\left|\Psi_{0}\right|$ up until high RH. Thus, $\Psi_{0}$ is about $-2 \mathrm{MPa}$ in $75 \mathrm{~nm}$ high channels as determined from the Kelvin equation, corresponding to a $\mathrm{RH}$ of $98.5 \%$, above which $\mathrm{RH}$ drying reverts into condensation [11]. The drying rate will be substantially $(>10 \%)$ reduced when $\left(\Psi_{e}\right)^{-3}$ becomes larger than $0.1\left(\Psi_{0}\right)^{-3}$ which is above $\mathrm{RH}=97 \%$.

The demonstrated phenomenon on the influence of sharp corners on drying can be of use for the optimal design of heat pipes, where the maximum heat transport is determined by the moment the wick dries out because of insufficient capillary flow [15]. Micromachined heat pipes and capillary-pumped loops that are applied for chip cooling generally employ microgrooves etched in silicon with corners of $70.5^{\circ}$ to guide capillary water [15-17]. Experimentally it has been shown that such grooves transport more water than rectangular ones and thus enable higher heat fluxes [18]. Grooves with sharper corners, however, have not been investigated. Design of microheat pipes with highly parallel arrays of sharp-cornered grooves would therefore drastically increase water transport before drying out would occur. Such groove arrays can, e.g., be micromachined using reactive ion etching [19].

The research was supported by the Dutch Ministry of Economic Affairs through a NanoImpuls Grant. J.E. wishes to thank Rogier Brussee for his assistance with the mathematics and Sebastiaan Herber for his assistance with the figures and his critical reading of the manuscript.

*On leave from Department of Physics, Indian Institute of Technology, Mumbai, India.

[1] M. Prat, Chem. Eng. J. (London) 86,153 (2002).

[2] A. G. Yiotis, A. K. Stubos, A. G. Boudouvis, and Y.C. Yortsos, Adv. Water Resour. 24, 439 (2001).
[3] N. Mao and S. J. Russel, J. Appl. Phys. 94, 4135 (2003); A. K. Haghi, J. Therm. Anal. 76, 1035 (2004).

[4] T. M. Shaw, Phys. Rev. Lett. 59, 1671 (1987).

[5] J. B. Laurindo and M. Prat, Chem. Eng. Sci. 53, 2257 (1998).

[6] G. Yiotis, A. G. Boudouvis, A. K. Stubos, I. N. Tsimpanogiannis, and Y.C. Yortsos, AIChE J. 50, 2721 (2004); A. G. Yiotis, A. G. Boudouvis, A. K. Stubos, I. N. Tsimpanogiannis, and Y.C. Yortsos, Phys. Rev. E 68, 037303 (2003).

[7] N. V. Churaev, Liquid and Vapor Flow in Porous Bodies: Surface Phenomena, Topics in Chemical Engineering (Gordon and Breach, New York, 2000), Vol. 13.

[8] J. C. T. Eijkel, J. G. Bomer, N. R. Tas, and A. van den Berg, Lab Chip 4, 161 (2004).

[9] N. R. Tas, J. Haneveld, H. V. Jansen, M. Elwenspoek, and A. van den Berg, Appl. Phys. Lett. 85, 3274 (2004).

[10] M. Tuller, D. Or, and L. M. Dudley, Water Resour. Res. 35, 1949 (1999).

[11] J.C. T. Eijkel and A. van den Berg, Lab Chip 5, 1202 (2005).

[12] A. W. Adamson, Physical Chemistry of Surfaces (Wiley, New York, 1967), 2nd Ed..

[13] M. Iwamatsu and K. Horii, J. Colloid Interface Sci. 182, 400 (1996).

[14] T. C. Ransohoff and C. J. Radke, J. Colloid Interface Sci. 121, 392 (1988).

[15] H. B. Ma and G. P. Peterson, J. Heat Transfer 120, 227 (1998).

[16] S.-W. Kang and D. Huang, J. Micromech. Microeng. 12, 525 (2002); M. Le Berre, S. Launay, V. Sartre, and M. Lallemand, J. Micromech. Microeng. 13, 436 (2003); R. H. Nilson, S. K. Griffiths, S. W. Tchikanda, and M. J. Martinez, J. Heat Transfer 126, 453 (2004).

[17] D. Trebotich, J. Kirshberg, J. Teng, and D. Liepmann, Modeling and Simulation of Microsystems (Nano Science and Technology Institute, Cambridge, MA, 2001), p. 262, ISBN 0-9708275-0-4.

[18] G. P. Peterson, A. B. Duncan, and M. H. Weichold, J. Heat Transfer 115, 751 (1993).

[19] M. J. de Boer, J. G. E. Gardeniers, H. V. Jansen, E. Smulders, M.-J. Gilde, G. Roelofs, J. N. Sasserath, and M. Elwenspoek, J. Microelectromech. Syst. 11, 385 (2002). 